\title{
Noise performance of the Herschel- SPIRE bolometers during instrument ground tests
}

Bernhard Schulz, James J. Bock, Nanyao Lu, Hien T. Nguyen, C. Kevin Xu, et al.

Bernhard Schulz, James J. Bock, Nanyao Lu, Hien T. Nguyen, C. Kevin Xu, Lijun Zhang, C. Darren Dowell, Matthew J. Griffin, Glenn T. Laurent, Tanya L. Lim, Bruce M. Swinyard, "Noise performance of the Herschel-SPIRE bolometers during instrument ground tests," Proc. SPIE 7020, Millimeter and Submillimeter Detectors and Instrumentation for Astronomy IV, 702022 (18 July 2008); doi: 10.1117/12.790163

SPIE Event: SPIE Astronomical Telescopes + Instrumentation, 2008, Marseille, France 


\title{
Noise Performance of the Herschel-SPIRE Bolometers during Instrument Ground Tests
}

\author{
Bernhard Schulz ${ }^{\mathrm{a}^{*}}$, James J. Bock ${ }^{\mathrm{b}}$, Nanyao Lu ${ }^{\mathrm{a}}$, Hien T. Nguyen ${ }^{\mathrm{b}}$, C. Kevin Xu ${ }^{\mathrm{a}}$, Lijun Zhang ${ }^{\mathrm{a}}$, \\ C. Darren Dowell ${ }^{\mathrm{c}}$, Matthew J. Griffin ${ }^{\mathrm{d}}$, Glenn T. Laurent ${ }^{\mathrm{e}}$, Tanya L. Lim ${ }^{\mathrm{f}}$, Bruce M. Swinyard $^{\mathrm{f}}$ \\ a Infrared Processing and Analysis Center, California Institute of Technology, MS 100-22, 1200 East \\ California Boulevard, Pasadena, CA 91125, USA; \\ ${ }^{b}$ Jet Propulsion Laboratory California Institute of Technology, 4800 Oak Grove Drive, Pasadena, \\ CA 91109, USA; \\ 'Division of Physics, Mathematics, and Astronomy, California Institute of Technology, MS 320-47, \\ 1200 East California Boulevard, Pasadena, CA 91125; \\ ${ }^{\mathrm{d}}$ Department of Physics and Astronomy, University of Wales, Cardiff, Queens Buildings, 5 The \\ Parade, Cardiff CF24 3YB, UK; \\ ${ }^{\mathrm{e}}$ Center for Astrophysics \& Space Astronomy, Univ. of Colorado, 389-UCB, Boulder, CO 80309, \\ USA; \\ ${ }^{\mathrm{f}}$ Rutherford Appleton Laboratory, Chilton, Didcot, Oxfordshire OX11 0QX, UK.
}

\begin{abstract}
The flight model of the SPIRE instrument underwent several test campaigns in a test facility at the Rutherford Appleton Laboratory (RAL) in the UK. A final dark campaign, completed in March 2007, provided an environment virtually free from optical radiation. This allowed re-determining the fundamental model parameters of the NTD spider web bolometer detector arrays in the new environment. The tests reported in this paper produced a fairly homogeneous dataset to investigate white noise and $1 / \mathrm{f}$ noise at different bias voltages, bias frequencies, and bath temperatures. We find that the white noise performance is in excellent agreement with the model predictions, once we correct the low frequency signal variations that are due to temperature fluctuations of the thermal bath at about $300 \mathrm{mK}$. The temperature of the thermal bath (detector array base plate) is measured by thermistor pixels that are part of the bolometer arrays. A residual 1/f component beyond those variations is hardly detected. This unexpected stability is very welcome and will positively impact photometer scan maps, the most popular observing mode of SPIRE.
\end{abstract}

Keywords: Herschel, space, instrumentation, far infrared, submillimeter, bolometer arrays

\section{INTRODUCTION}

The Spectral and Photometric Imaging Receiver (SPIRE) is one of three scientific instruments carried on board of the Herschel Space Observatory. Herschel is an ESA mission with NASA participation that will explore the Universe at FarInfrared and Submillimeter wavelengths. SPIRE is being designed and built by a consortium of institutes and university departments from across Europe, Canada, China, and the USA, under the leadership of a Principal Investigator (Professor M.J. Griffin) located at the University of Wales, Cardiff, UK [1]. Consisting of a photometer and a spectrometer section, SPIRE covers a wavelength range of 194 to $672 \mu \mathrm{m}$. It is designed to address important astrophysical questions of galaxy formation and star formation through deep photometric imaging surveys in 3 photometric bands centered at 250, 350 and $500 \mu \mathrm{m}$. Follow-up observations of individual sources can be made with its imaging Fourier Transform Spectrograph (FTS) that offers variable spectral resolution between 15 and 1289, depending on wavelength and observing mode.

With a "cool" but not "cold" $80 \mathrm{~K}$ telescope mirror, the noise from its thermal photon background fundamentally limits the sensitivity. NTD spider web bolometers [6] were chosen as detectors for both sub-instruments. They are arranged in

\footnotetext{
*e-mail: bschulz@ipac.caltech.edu; phone: +1 626 395-1874; fax +1 626 3970-7354
}

Millimeter and Submillimeter Detectors and Instrumentation for Astronomy IV edited by William D. Duncan, Wayne S. Holland, Stafford Withington, Jonas Zmuidzinas Proc. of SPIE Vol. 7020, 702022, (2008) · 0277-786X/08/\$18 · doi: 10.1117/12.790163 
three arrays of $43(500 \mu \mathrm{m}), 88(350 \mu \mathrm{m})$ and $139(250 \mu \mathrm{m})$ detectors, which are coupling to the telescope via hexagonally close-packed $2 \mathrm{~F} \lambda$-diameter single-mode conical feedhorns, giving diffraction limited beams of FWHM 18, 25 and 36" respectively. Two beam splitters allow for simultaneous observation in all three wavelength bands [1]. The bolometer detector assemblies (BDA) and the JFET pre-amplifiers were manufactured at the Jet Propulsion Laboratory (JPL) in the US, and, after performance testing [3][4], shipped to the Rutherford Appleton Laboratory (RAL) in the UK for integration into the instrument.

\section{METHODOLOGY}

In this paper we report on the verification of the noise performance of the SPIRE detector arrays. The Proto Flight Model (PFM) was subjected to a total of 5 test campaigns in a calibration facility [7] at RAL. The last one, called PFM 5, was performed in virtual dark conditions with the optical window of the cryostat sealed-off. The previous tests had shown more background radiation entering through the neutral filters from the laboratory than expected that also showed temporary variations. We opted for a final verification of detector performance in an optically sealed cryostat that would ensure stable and well understood test conditions. In particular the noise performance results are readily compared with predictions by theory using the parameters determined during the assembly level tests at JPL. We follow here the formulae as described by Nguyen et al. [9]. The bolometer model, validated in this way, can then provide the required sensitivities at in-flight background levels.

\section{EXPERIMENT DETAILS}

\subsection{The Signal Chain}

In the following we will give a brief overview over the electronic layout of the SPIRE signal chain. It is the same for both photometer and spectrometer detectors, although parameters like bias frequency, bandpass- and lowpassfrequencies, and amplifications factors are different. A graphic representation can be found in Fig. 1.

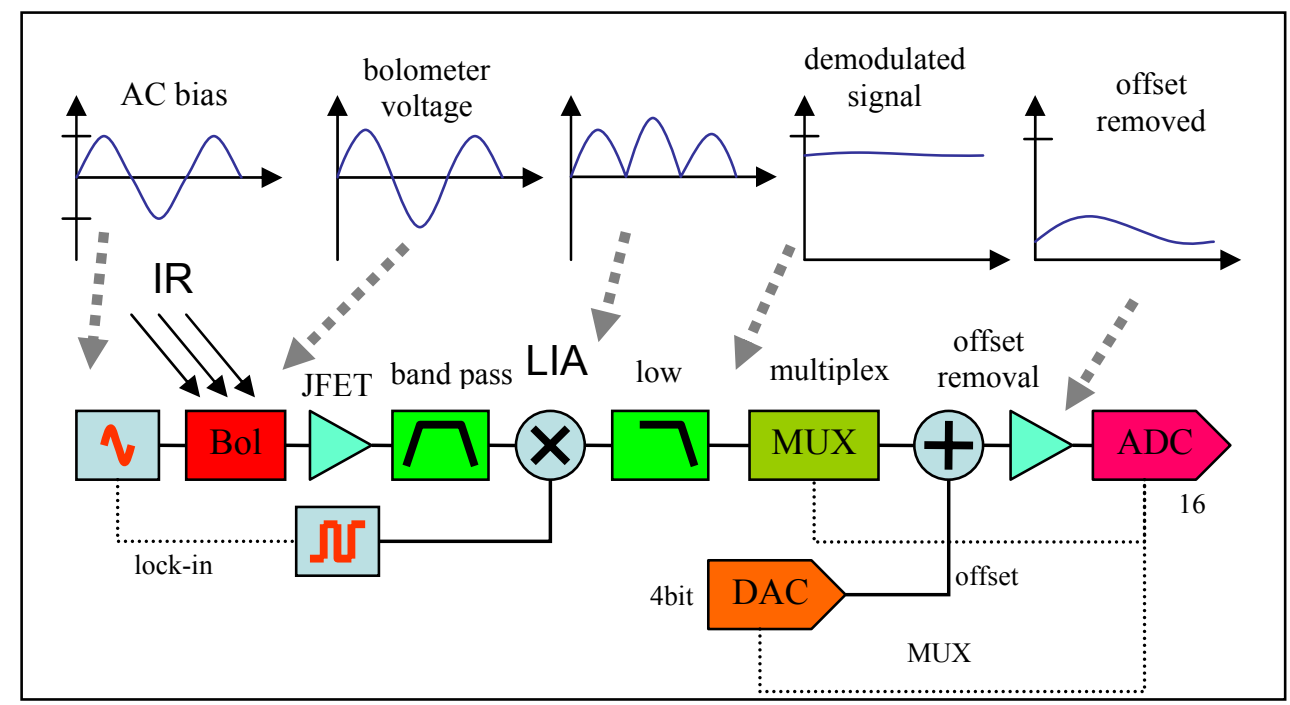

Fig. 1. Schematic diagram of the SPIRE signal chain. The AC bias induces a sine wave bolometer voltage that is amplified by a JFET and demodulated by a lock-in amplifier (LIA). Bolometer and JFET are located within the cryostat. The multiplexer stage also removes a 4-bit offset to adapt to the large practically constant thermal signal from the telescope.

A sine wave AC-bias that can be selected between 50 and $300 \mathrm{~Hz}$ is applied to the bolometer that is connected symmetrically in line with two load resistors. The bolometer voltage is pre-amplified by JFET amplifiers. They are mounted inside the cryostat close to the instrument at the $10 \mathrm{~K}$ level of the optical bench and internally heated to their optimum operating temperature of $\sim 120 \mathrm{~K}$. The first warm pre-amplifier of the warm electronics includes a bandpass filter that reduces electromagnetic noise picked up by the long harness on the way from inside the cryostat. The frequency ranges are 33-1312 Hz and 33-2414 $\mathrm{Hz}$ for photometer and spectrometer respectively [9]. This is followed by a Lock-In Amplifier (LIA) consisting of a multiplier stage and a lowpass filter. The signal is first multiplied with a biassynchronized square-wave signal whose phase can be shifted w.r.t the bias. This is followed by a 4-pole Bessel low-pass 
filter with cut off frequency of $4.98 \mathrm{~Hz}$ for the photometer or a 6-pole Bessel low-pass filter with cut off frequency of $24.44 \mathrm{~Hz}$ for the spectrometer. The following multiplexer and analog to digital conversion stage removes an individual constant offset voltage from each channel. This is to eliminate the large fraction of the signal originating in the thermal emission of the telescope mirror. The offset is controlled by a 4-bit D/A converter and typically set automatically at the beginning of a measurement.

\subsection{The Bolometer Array Configuration}

The spider web bolometer arrays for SPIRE have been described already in the literature [10], but for the sake of the following discussion we will point out a few details here again. Figure 2 shows a picture of the $500 \mu \mathrm{m}$ bolometer array (PLW) without the feedhorn block. The bolometer pixels are arranged in a hexagonal pattern across the base plate and the symmetric load resistor arrays are mounted on both sides of it. In addition there are two dark pixels, which are regular bolometer pixels mounted between feedhorns where they receive no light, although small light leakage up to $0.5 \%$ from neighboring pixels is possible [10]. Thermistor pixels are available too to provide a means of measuring the temperature of the base plate very close to the bolometers. One more channel is connected to a resistor instead of a bolometer. All channels are biased by the same AC source.

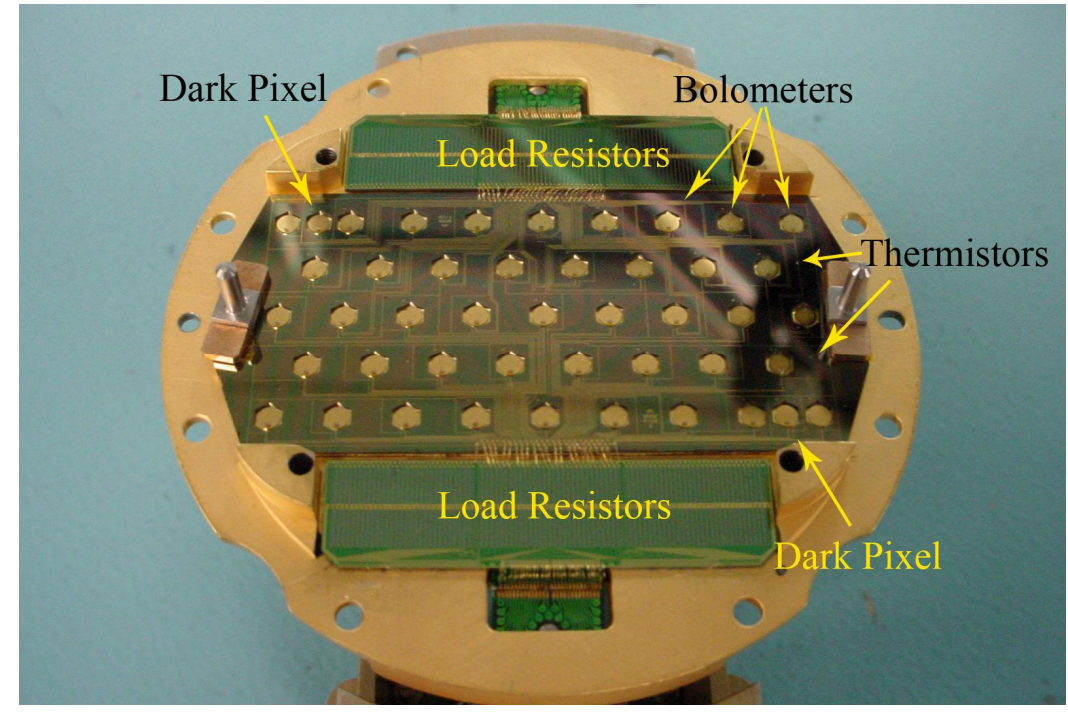

Fig. 2. Picture of the $500 \mu \mathrm{m}$ bolometer array (PLW) without the feedhorn block obscuring the view. Locations of dark pixels, thermistor pixels, a few exemplary bolometer pixels and the load resistor arrays are indicated.

\section{MEASUREMENTS AND DATA REDUCTION}

\subsection{Measurement of the JFET Noise}

Each JFET module comprises 24 detector channels. For each module an optimum bias can be selected. The correct choice of the JFET bias voltage is not critical, but should be part of the initial setup of the system to achieve the right working temperature for the JFETs. Otherwise spontaneous spiking and excess noise can occur, as observed in test campaigns prior to PFM 5. To optimize the operating point of the JFET pre-amplifiers, the pure amplifier noise was measured with the bolometers at temperatures of $\sim 4 \mathrm{~K}$. At this temperature the resistance of the bolometers is negligible compared to that of the load resistors, and thus the bolometer noise. The electronic signal under these circumstances is very stable and the white noise can be determined simply from the white noise plateau of the power spectrum as described below. The JFET bias was varied over a range in 11 steps for both photometer and spectrometer and the noise was plotted against JFET bias voltage. The optimum JFET bias was determined for each module seeking a balance between minimum and uniform noise for all 24 channels of a module as well as a minimum in power consumption. We find an average optimized amplifier noise level of 8.9 and $7.9 \mathrm{nV} \mathrm{Hz}^{-1 / 2}$ for photometer and spectrometer respectively, well beyond the requirement of $15 \mathrm{nV} \mathrm{Hz}^{-1 / 2}$ and stopping short of the goal of $7 \mathrm{nV} \mathrm{Hz}^{-1 / 2}$. 


\subsection{Noise Measurements at different Bias Voltages and Temperatures}

To optimize the detector noise performance, determine the influence of temperature and bias frequency, and compare the data with the model predictions, a noise data cube was measured. Dark noise measurements were carried out at two temperatures of the $\mathrm{He}^{3}$ fridge at 286 and $297 \mathrm{mK}$, under a range of bias frequencies at 80-201 $\mathrm{Hz}$ for the photometer and $100-305 \mathrm{~Hz}$ for the spectrometer, and typically 10 to 12 peak bias amplitudes in the range of 0-86.6 $\mathrm{mV}$ for the photometer and 4.8-171.5 mV for the spectrometer. The PFM 5 data analyzed here cover a total of 103 and 96 points in this parameter space for the photometer and spectrometer, respectively.

The resistances of bolometer and load resistors together with the stray capacitances between bolometer and JFET form an RC filter and introduce a phase shift to the signal. Since the bolometer resistance changes significantly under all these different configurations, the resulting phase shift varies. To avoid signal losses in the LIA, the necessary phase shift between the sine wave from the bias source and the square wave feeding the LIA was pre-determined through "phaseup" measurements for each parameter combination and applied during the measurement sequence.

\subsection{Noise Data Processing}

The recorded detector voltages were processed using an IDL-based bolometer analysis software package originally developed at IPAC [5]. Each observation is divided into individual timelines for which parameters like bias, bias frequency, offset, and temperature are constant. Each timeline spans about 500 seconds. The raw signal and the offset values are converted to voltage measured at the detector output and co-added. This takes into account the known frequency dependent amplification factors along the signal chain. For the JFET pre-amplifiers a fixed amplification factor of 0.96 was assumed since the actually measured factors are very uniform. We note that we did not apply a correction for stray capacitances that exist between the bolometers and the JFETs and are of the order of $50 \mathrm{pF}$. The corrections are not yet conclusively determined, but are estimated to be below per-cent level for the bias frequency of 70 $\mathrm{Hz}$ that we used in our measurements. For higher bias frequencies the effect on the measured temperatures of the thermistor pixels could become significant and will need further investigation. A correction for phase is not necessary as each parameter combination was individually phased-up as mentioned above.

Signals were plotted versus time and visually inspected. Typically the signal contained variations that we found corresponding to small temperature changes of the bolometer base plate, which is equivalent to the bath temperature $T_{0}$ (see [3]) in the bolometer model. As this correction is quite important, it is described in more detail in the next chapter and we note that we apply it at this stage of the process. If not applied, the resulting $1 / \mathrm{f}$ knee starts at much higher frequencies

After temperature drift correction, the signal was de-glitched and further divided into 100 sec segments corresponding to a lower limiting frequency of $0.01 \mathrm{~Hz}$. These segments were each Fourier transformed, normalized and then quadratically co-added to yield the final power spectrum for a given channel. For each power spectrum, a white noise plateau $\sigma$ was determined as the median value between 0.5 and $2.0 \mathrm{~Hz}$. The power spectrum at lower frequencies was fit to the sum of $\sigma$ and a $1 / \mathrm{f}$ component. The frequency where the two components equal each other is identified as the $1 / \mathrm{f}$ knee frequency $f_{\text {knee }}$.

\section{TEMPERATURE CORRECTION}

\subsection{Signal Dependence on $\mathbf{T}_{0}$}

We generally observe long term signal drifts in bolometer signals recorded over several hours. The signals of the thermistor and dark pixels follow these trends, while the signal of the resistor pixel is very stable. As the thermistor pixels measure the bath temperature $T_{0}$, this suggests that a significant portion of the drift is not intrinsic $1 / \mathrm{f}$ noise of the bolometer but temperature related.

To quantify these drifts, we analyzed longer datasets of the PFM 5 campaign that where taken during presumably quiet times over night with constant parameter settings. The scale of the signal drift is on the order of $0.1 \%$, though occasionally it can be as high as $\sim 0.5 \%$ of the bolometer voltage. Plotting bolometer signals versus the signal of one of the local thermistor pixels of a BDA shows an almost perfect correlation (see Figure 3 for some examples). 

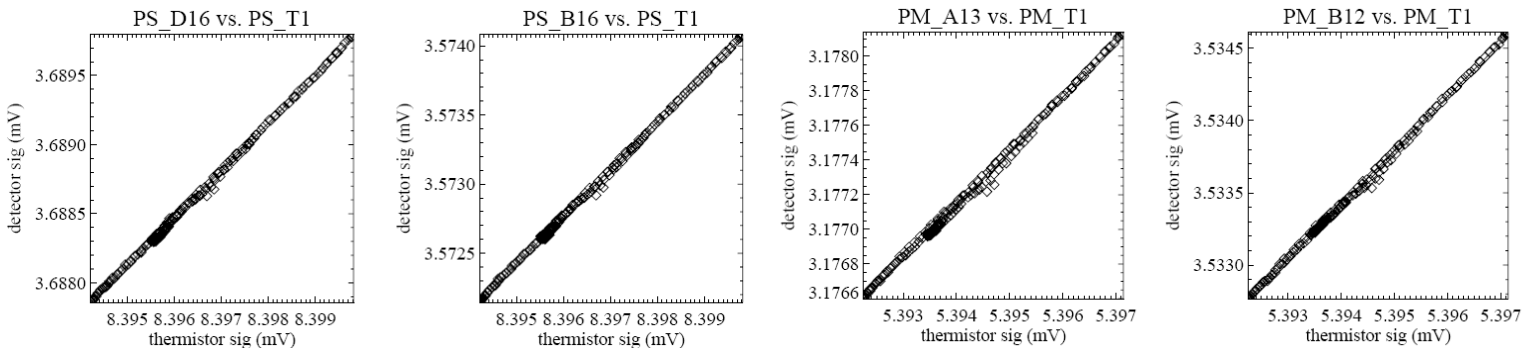

Fig. 3. Bolometer signals plotted versus thermistor pixel signals. The correlation is very strong.

\subsection{Temperature Correction Methods}

The very tight linear correlation suggests an empirical approach to correct the signal for temperature drift. We assume (i) the drift of the bath temperature is proportional to the drift of the thermistor signals, and (ii) the tight detector/thermistor correlation specifies the temperature dependence of the detector signal. These assumptions are valid only when the amplitude of the signal drift is much less than the signal itself (small perturbation condition), which generally applies in our case.

The two elements of our temperature correction method are (i) a smoothing algorithm that removes the non-thermal high frequency component from the thermistor signal, and (ii) a function that corrects the signals of the bolometer pixels depending on the smoothed thermistor signals in such a way that the average of each bolometer signal stays the same.

If we define the drift signal $\mathrm{S}_{\text {drift }}=\mathrm{S}-<\mathrm{S}>$, where $\mathrm{S}$ is the vector of time ordered signal data of a thermistor pixel or a bolometer pixel, and $<\mathrm{S}>$ is the average of these values, we can define the smoothing functions $\mathrm{f}_{\mathrm{T} 1}\left(\mathrm{~S}_{\mathrm{driff}}\right)$ and $\mathrm{f}_{\mathrm{T} 2}\left(\mathrm{~S}_{\text {drift }}\right)$ to be the smoothing functions of the T1 and T2 thermistor pixels respectively. We have tested (i) a $3^{\text {rd }}$ order polynomial, (ii) a spline fit, and (iii) a moving median with different time intervals resulting in 5 different smoothing algorithms listed in Table 1.

Table 1. List of algorithms to fit the thermistor signal by a smoothing function.

\begin{tabular}{lr|l|l}
\multicolumn{1}{c|}{ Name } & Function form & $\Delta \mathbf{t}$ & Note \\
\hline CUBIC & $3^{\text {rd }}$ order polynomial & 5.0 & Fit to Time ordered Data $($ ToD) of $600 \mathrm{sec}$ \\
SPLIN5 & SPLINE & 5.0 & SPLINE fit to ToD smoothed to $\Delta \mathrm{t}=5.0 \mathrm{sec}$ \\
SPLIN1 & SPLINE & 1.0 & SPLINE fit to ToD smoothed to $\Delta \mathrm{t}=1.0 \mathrm{sec}$ \\
MMD10 & Moving Median & 1.0 & Moving median of ToD with tine window $\Delta \mathrm{t}=1.0 \mathrm{sec}$ \\
MMD03 & Moving Median & 0.3 & Moving median of ToD with time window $\Delta \mathrm{t}=0.3 \mathrm{sec}$
\end{tabular}

The correction algorithm can be written as: $S_{\text {corr }}=S-\left(a_{0}+a_{1}\left(a_{2} f_{T 1}+a_{3} f_{T 2}\right)\right)$. We tested 4 configurations of this formula, by fixing selected parameters $\mathrm{a}_{\mathrm{i}}$ to be 0 or 1 , detailed in Table 2 . The other parameters, designated "cal" in the table are determined from fits to a diagram like the ones shown in Figure 3.

Table 2. List of algorithms that were tested to correct temperature dependent bolometer signal variations (see text).

\begin{tabular}{l|l|l|l|l|l} 
Name & $\mathbf{a}_{\mathbf{0}}$ & $\mathbf{a}_{\mathbf{1}}$ & $\mathbf{a}_{\mathbf{2}}$ & $\mathbf{a}_{\mathbf{3}}$ & Method Description \\
\hline T1 & cal & 1 & cal & 0 & Using thermistor T1 only for correction. \\
T2 & cal & 1 & 0 & cal & Using thermistor T2 only for correction. \\
T1 + T2 & cal & cal & 1 & 1 & Using the sum of both thermistor signals to correct. \\
T1 \& T2 & cal & 1 & cal & cal & Using a weighted sum of the thermistor pixels to correct.
\end{tabular}

\subsection{Optimization}

The $1 / \mathrm{f}$ knee frequency in the power spectrum comes first to mind to be used as a metric, when comparing the correction efficiency of all these combinations. However, we found that our method was so efficient, that the lower frequency cut 
off at $\sim 10 \mathrm{mHz}$, that we chose to achieve a better $\mathrm{S} / \mathrm{N}$ on the spectra, made it difficult to determine whether a $1 / \mathrm{f}$ rise was still present within that range. At the lowest frequencies the power spectrum, even after co-adding, remains relatively noisy due to lack of data. Instead, a more robust indicator was used: the ratio between the median over a defined interval in the low frequency power spectrum $(3 \ldots 30 \mathrm{mHz})$ and the white noise level (median over 0.5..2.0 Hz). We find a good correlation between this ratio and the results from the automatic $1 / \mathrm{f}$ knee frequency analysis. However, we will have to analyze longer duration datasets to determine the general shape of the power spectrum after temperature drift correction and where the new frequency of a $1 / \mathrm{f}$ knee might be.

In any case the results of this exercise are very encouraging. After temperature drift correction we can generally not identify a $1 / \mathrm{f}$ knee anymore above $50 \mathrm{mHz}$, except for a few noisy pixels. It appears that the T1\&T2 method yields the best results and the $\mathrm{T} 1+\mathrm{T} 2$ method the worst. However, judging from the median residual low frequency noise, the difference between the methods is only $\sim 4 \%$. Nevertheless, for individual detector channels, the difference can be as large as $20 \%$. Among the 5 smoothing algorithms studied here, CUBIC cannot handle temperature drifts well, that are on a time scale shorter than a few tens of seconds. On the other hand, the CUBIC correction results in the lowest residual low frequency noise for data without sharp temperature fluctuations. The moving median algorithm MMD03 doesn't suppress high frequency white noise enough so that it becomes added to the corrected detector signal. The two algorithms based on the SPLINE fit function give the most robust performance. In Figure 4 examples of uncorrected (left) and corrected (right) signal timelines are shown.
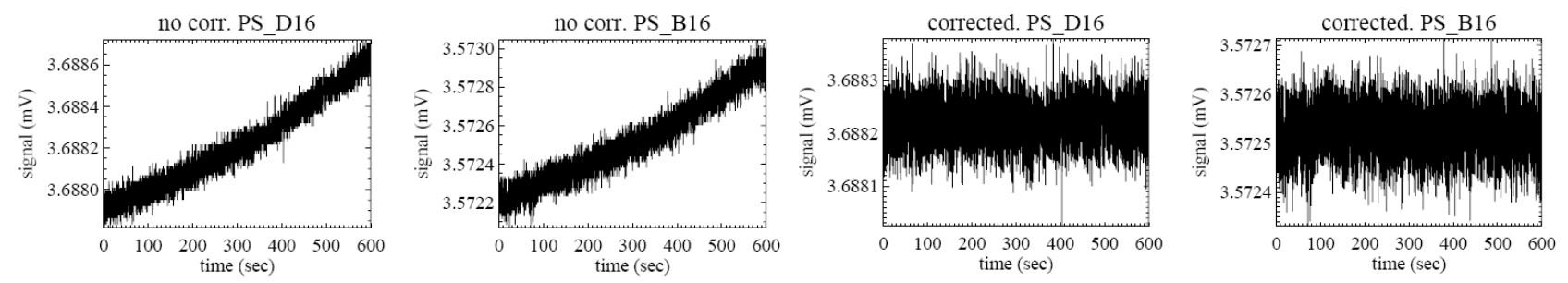

Fig. 4. Examples of two detector signal timelines before (left two diagrams), and after (right two diagrams) using SPLIN5 smoothing with $\mathrm{T} 1$ for correction. The detector pixels are part of the photometer array PSW centered at $250 \mu \mathrm{m}$.

\section{RESULTS}

We removed the thermal drift empirically as described in the previous chapter using the signals of both thermistor pixels (T1+T2 method, except for PMW with the T1 method $)^{\dagger}$ and a spline smoothing with a time interval of 5 sec before a noise analysis was done. This removed the $1 / \mathrm{f}$ knee contributions to a large extent.

Due to the temperature gradient along the thermal strap that leads to the BDAs the actual bolometer bath temperatures are higher. With the thermistor pixels we measure photometer temperatures of 328 and $339 \mathrm{mK}$ and spectrometer temperatures of 301 and $316 \mathrm{mK}$ at fridge temperatures of 286 and $297 \mathrm{mK}$ respectively. The error is estimated to be \pm 3 $\mathrm{mK}$ and is dominated by systematic uncertainties in the calibration.

We compared the measured noise $\sigma$ directly with that derived from the bolometer model [3] and find remarkably good agreement for the photometer arrays and still reasonably good agreement for the spectrometer arrays. Figure 5 shows histograms of the ratios between measured and modeled noise for all detectors of the photometer arrays (left) and the spectrometer arrays (right) at the lower fridge temperature. The majority of the photometer data points are found within $10 \%$ of the model while the majority of the spectrometer data points are found within $15 \%$ on the model.

With the bolometer responsivity $\mathrm{S}$, calculated by the model, we can derive the noise equivalent power $\mathrm{NEP}=\sigma / \mathrm{S}$. In Figure 6 on the left we show an example of a diagram, in this case for the PLW that was produced for every detector array. It shows the array medians of the NEP, calculated disregarding dead and exceptionally noisy detectors, plotted versus peak amplitude detector bias. The black and white symbols designate data measured at the two temperature levels that form two distinct curves with clear and distinct minima as expected.

\footnotetext{
${ }^{\dagger}$ The noise data processing preceded the results from the optimization analysis, but the difference to the results of a T1 \& $\mathrm{T} 2$ processing would be very small and would not change the results significantly.
} 

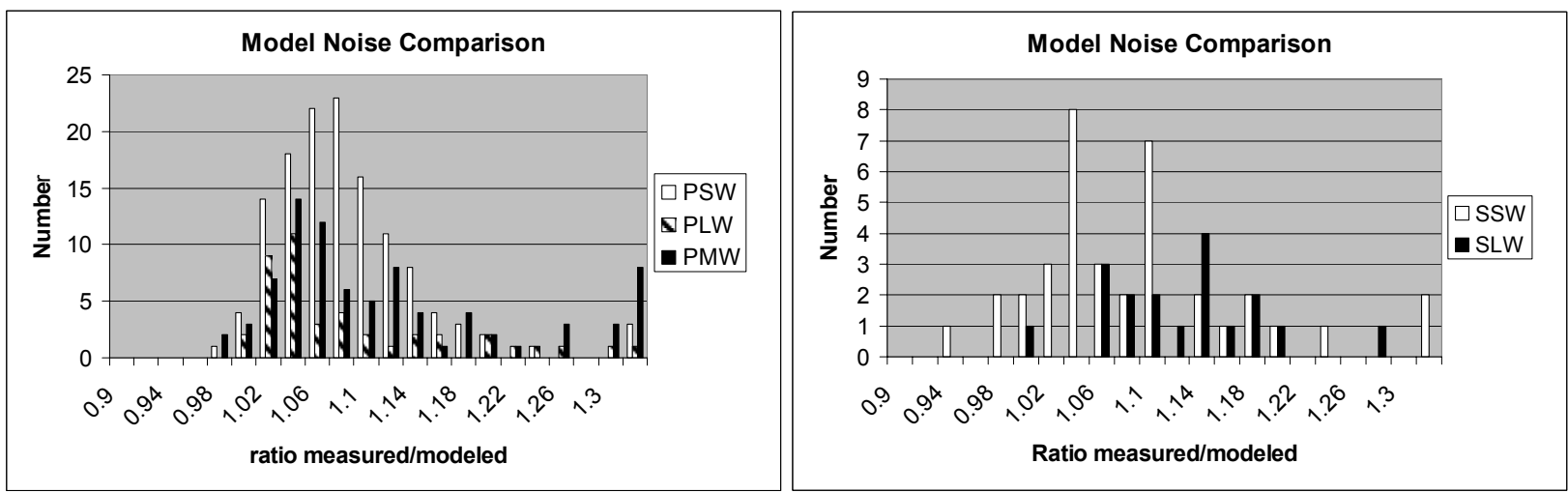

Fig. 5. Distribution of ratios of measured and modelled dark noise values for photometer (left) and spectrometer (right).

The right diagram of Figure 6 shows the same data points plotted as ratio of measured and modeled noise versus bias voltage, providing another direct comparison with the model. For biases above $10 \mathrm{mV}$ the PLW data show excellent consistency with the model within a $\pm 10 \%$ range. The different symbols indicate bias frequency as explained in the figure caption. Their distribution, showing the higher noise values for lower frequencies, may be due to the fact that we do not correct for signal losses due to stray capacitances. The lower $\mathrm{He}^{3}$ fridge temperature of $286 \mathrm{mK}$ led to lower noises and a lower optimum bias voltage in accordance with the model.
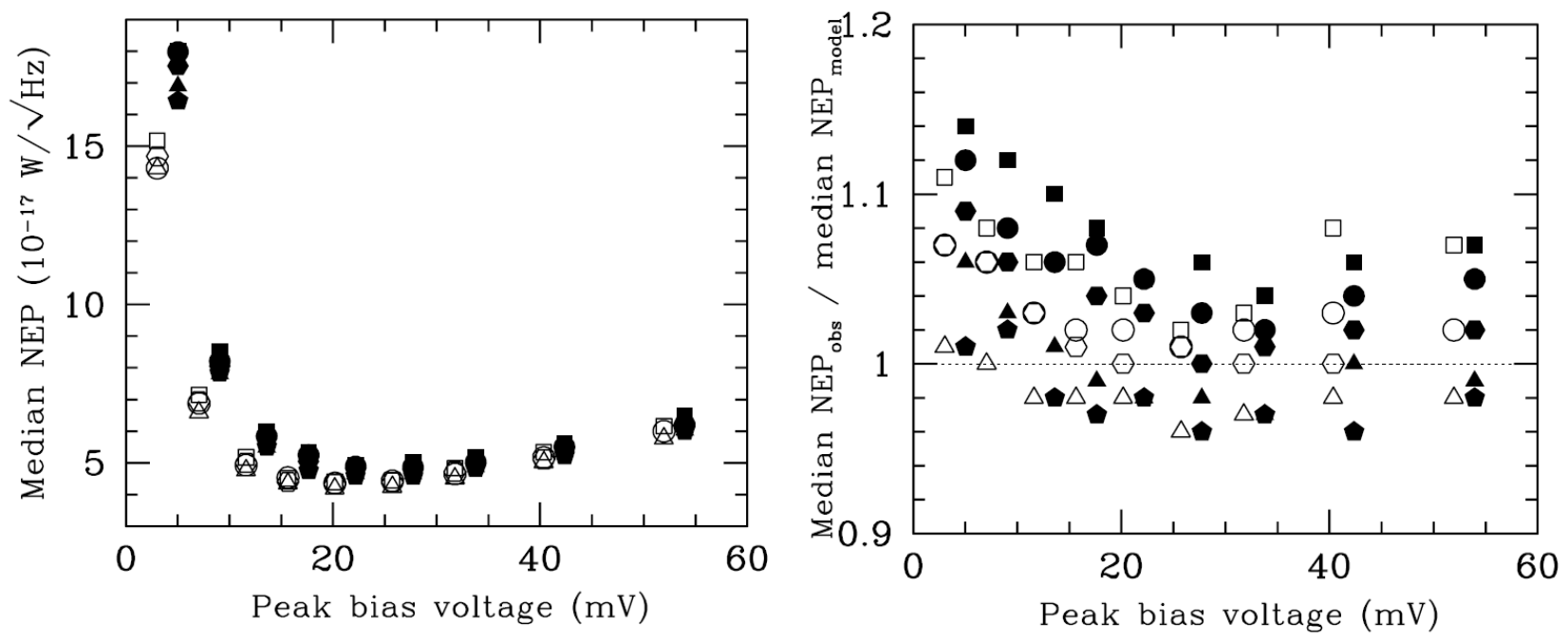

Fig. 6. Example median NEP values of the PLW array $(500 \mu \mathrm{m}$ center wavelength) for different bias levels and bias frequencies (left). The right diagram shows the comparison with the bolometer model. The colors indicate $\mathrm{He}^{3}$ fridge temperature (white $=286 \mathrm{mK}$; black=297 mK). The symbols indicate bias frequencies: $80.4 \mathrm{~Hz}$ (squares), $110.4 \mathrm{~Hz}$ (circles), $131.1 \mathrm{~Hz}$ (hexagons), $171.3 \mathrm{~Hz}$ (triangles) or $201.4 \mathrm{~Hz}$ (pentagons). For the purpose of clarity, there is a slight horizontal offset between the black and white symbols.

What we find for the PLW array in Figure 6 applies also to the other two photometer arrays PSW and PMW. We find excellent agreement with the model within $10 \%$, especially at biases $>10 \mathrm{mV}$. The NEP minima for the photometer arrays under dark conditions are located at $\mathrm{V}_{\text {bias }}=20$ to $25 \mathrm{mV}$. The array median NEPs at the optimal $\mathrm{V}_{\text {bias }}$ reach minima at about $4.6,4.6$, and $4.3 \cdot 10^{-17} \mathrm{~W} \mathrm{~Hz}^{-1 / 2}$ for PSW, PMW and PLW, respectively.

For the spectrometer, the overall agreement between measurement and model is still good, but individual measurements like the one at $100 \mathrm{~Hz}$ bias frequency is larger than for the photometer. We noticed that another spectrometer measurement using the same bias frequency of $100 \mathrm{~Hz}$ on a different date shows a much better agreement with the model prediction. It is possible that additional noise sources or instability may have contributed. In some measurements the agreement between measurement and model is as good as $5 \%$, but the scatter is quite large. Around the optimal bias voltage of $\sim 35 \mathrm{mV}$, most of the measurements agree with the model predictions to about $10-15 \%$. In spite of the scatter 
in the spectrometer data, the data points in the respective diagrams of NEP versus bias still form a well-defined frontier consisting of all the minimum NEPs at the bias voltages used. The minimum of this frontier occurs at $\mathrm{V}_{\text {bias }} \sim 35 \mathrm{mV}$ for both SSW and SLW. At this optimal bias voltage under dark conditions, the median array NEPs reach a minimum of about $6 \cdot 10^{-17} \mathrm{~W} \mathrm{~Hz}^{-1 / 2}$ for both SSW and SLW.

Figure 7 shows the distribution of all dark NEPs for the photometer (left) and spectrometer arrays (right) at $286 \mathrm{mK}$. Both distributions are nicely localized, with those of the spectrometer being somewhat wider. The distribution maxima are found to be at about 4.6, 5.0, 4.8, 6.2 , and $6.2 \cdot 10^{-17} \mathrm{~W} \mathrm{~Hz}^{-1 / 2}$.
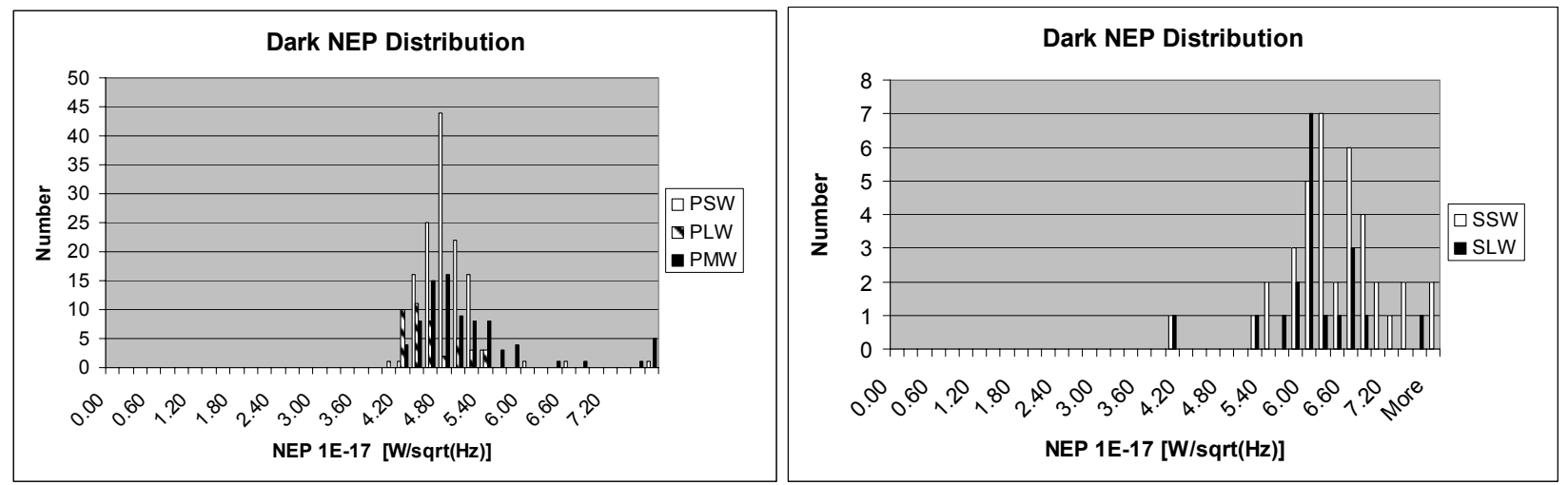

Fig. 7. Distribution of Dark NEP values for photometer bolometers (left) and spectrometer bolometers (right) at the lower fridge temperature of $286 \mathrm{mK}$.

\subsection{Optimum In-Flight Bias}

The noise parameters established from PFM 5 measurements apply strictly only to dark conditions. Due to the $\sim 80 \mathrm{~K}$ telescope mirror, the detectors will experience a substantial optical load during the mission. The good agreement of the model predictions with the measured noise data under dark conditions has shown its general applicability and reliability. We used it to predict the in-flight optimum bias values. Since the expected telescope emissivity is predicted to be considerably lower than what was assumed at the time the instrument was designed, we used corresponding in-band optical loads of 1.7, 1.0, 1.2, 4.1, and $4.9 \mathrm{pW}$ for PSW, PMW, PLW, SSW, and SLW respectively. We used bath temperatures of $330 \mathrm{mK}$ for the photometers and $296 \mathrm{mK}$ for the spectrometers, which are temperatures that were typical for the non-heated tests during PFM 5. Under optically loaded conditions in-flight, the model predicts optimum bias voltages that are slightly higher than in the dark by $2-10 \mathrm{mV}$. The background limited NEPs should increase by $43 \%$, $20 \%, 23 \%, 43 \%$, and $51 \%$ for PSW, PMW, PLW, SSW, and SLW respectively compared to the dark NEPs.

\section{CONCLUSION}

The noise performance of the SPIRE bolometer arrays at instrument level under dark conditions corresponds within 10 to $15 \%$ to the model predictions. This confirms the sensitivity predictions for Herschel-SPIRE made by the uplink software HSPOT that is based on the same model, and is used for observation proposal generation. We also found an empirical method to correct temperature dependent long term signal variations. The corrected signals show no $1 / \mathrm{f}$ knee above $50 \mathrm{mHz}$, which will significantly improve data quality and particularly benefit the important large area scan observing mode of the SPIRE instrument.

Acknowledgement: The authors would like to thank the staff at RAL for their support in running the calibration facility and obtaining the data. Funding for this work was provided by the Planck and Herschel Project at the Jet Propulsion Laboratory and the NASA Herschel Science Center at IPAC/Caltech.

\section{REFERENCES}

[1] M. Griffin, A. Abergel, P. Ade, P. André, J-P. Baluteau, J. Bock, A. Franceschini, W. Gear, J. Glenn, D. Griffin, K.King, E. Lellouch, D. Naylor, G. Olofsson, I. Perez-Fournon, M. Rowan-Robinson, P. Saraceno, E. Sawyer, 
A.Smith, B. Swinyard, L. Vigroux, and G. Wright, "Herschel-SPIRE: Design, Performance, and Scientific Capabilities", Proc. SPIE 6265, 241-248 (1997).

[2] Anthony D. Turner, James J. Bock, Jeffrey W. Beeman, Jason Glenn, Peter C. Hargrave, Viktor V. Hristov, Hien T. Nguyen, Faiz Rahman, Srinivasan Sethuraman, Adam L. Woodcraft, "Silicon nitride Micromesh Bolometer Array for Submillimeter Astrophysics", Applied Optics, Vol. 40, Issue 28, pp. $4921-4932$ (Oct 2001)

[3] H.T. Nguyen, J.J. Bock, P. Ringold, J. Battle, S.C. Elliott, A.D. Turner, M. Weilert., V. V. Hristov, B. Schulz, K. Ganga, L. Zhang, J.W. Beeman, P.A.R. Ade, P.C. Hargrave, "A Report on Laboratory Performance of the Spectroscopic Detector Arrays for SPIRE/HSO," Proc. SPIE 5498, 196-207 (2004).

[4] H.T. Nguyen, P. Ringold, P.A.R. Ade, J. Battle, J.W. Beeman, J.J. Bock, S.C. Elliott, P.C. Hargrave, B. Schulz, A.D. Turner, V.V. Hristov, M. Weilert, L. Zhang, "A Report on Laboratory Performance of the Bolometric Detector Arrays for SPIRE/HSO (paper II)," Proc. SPIE 6275, 627518 (2006).

[5] Schulz, B., Zhang, L., Ganga, K., Nguyen, H., \& Holmes, W., "An Analysis Package for Bolometer Ground Testing, " ASP Conf. Series V.347, P. L. Shopbell, M. C. Britton, and R. Ebert eds. , p. 158 (2005).

[6] James J. Bock, Jason Glenn, Sabrina M. Grannan, Kent D. Irwin, Andrew E. Lange, Henry G. LeDuc, A.D. Turner, "Silicon nitride micromesh bolometer arrays for SPIRE," SPIE 3357, 297-304 (1998)

[7] Patrick A. Collins, P. A. R. Ade, M. Caldwell, M. Ferlet, M. J. Griffin, P. C. Hargrave, M. R. Harman, D. L. Smith, B. M. Swinyard, "A Ground Calibration Facility for HERSCHEL-SPIRE, " SPIE 4850, 628-637 (2003)

[8] Tanya Lim, Bruce Swinyard, Matthew Griffin, Asier Aramburu, Jean-Paul Baluteau, James Bock, Marc Ferlet, Trevor Fulton, Douglas Griffin, Steven Guest, Peter Hargrave, Kenneth King, Sarah Leeks, David Naylor, Edward Polehampton, Davide Rizzo, Eric Sawyer, Bernhard Schulz, Sunil Sidher, Locke Spencer, David Smith, Hien Trong Nguen, Ivan Valtchanov, Tim Waskett, Adam Woodcraft, "Preliminary Results From Herschel-SPIRE Flight Instrument Testing, " SPIE 6265, 62650F, 1-10 (2006)

[9] F. Pinsard, C. Cara, "HERSCHEL/SPIRE Detector Control Unit Design document", SPIRE-SAP-PRJ-001243, internal document 11/07/2005

[10] B. Rownd, J.J. Bock, G. Chattopadhyay, J. Glenn, and M. Griffin, "Design and performance of feedhorn-coupled bolometer arrays for SPIRE," SPIE 4855, 510-519 (2003) 